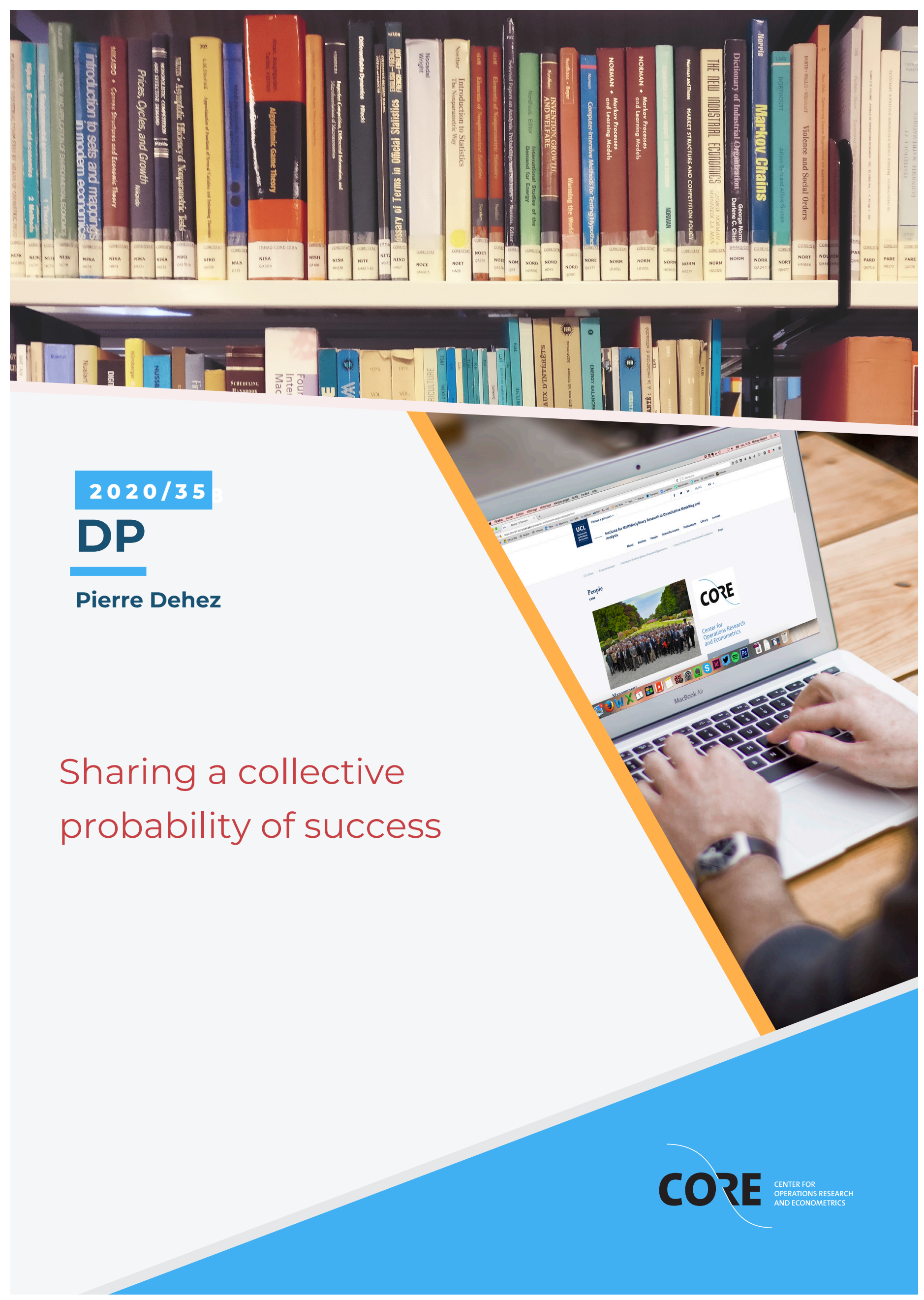




\section{CORE}

Voie du Roman Pays 34, L1.03.01

B-1348 Louvain-la-Neuve

Tel (32 10) 474304

Email: immaq-library@uclouvain.be

https://uclouvain.be/en/research-institutes/lidam/core/core-discussion-papers.html 
CORE Discussion Paper 2020/35

Revised March 2023

To appear in Mathematical Social Sciences

\title{
Sharing a collective probability of success
}

\author{
Pierre Dehez \\ Center for Operations Research and Econometrics (CORE-LIDAM) \\ University of Louvain, Louvain-la-Neuve \\ pierre.dehez@uclouvain.be
}

\begin{abstract}
How to allocate the probability of reaching a target resulting from the joint action of a group of players? This question is framed within transferable utility games. We analyze the properties of games resulting from different scenarios, characterize their core and provide a proper axiomatic foundation to their Shapley value.
\end{abstract}

Keywords: probability games, core, Shapley value JEL Classification: C71

* The author is grateful to Manfred Besner, Samuel Ferey, Joaquin Sanchez-Soriano, Pier Mario Pacini and two referees for useful comments and suggestions on earlier drafts. Special thanks go to Françoise Forges for her support and timely advice. 


\section{Introduction}

We consider situations where different actors have a common target to reach, knowing the probabilities with which actors will succeed in attaining the target. The question concerns the determination of the share of each actor in the collective probability of success defined as the probability that at least one actor attains the target. The resulting shares can then eventually be used for allocative purposes. This could be applied to various domains: financial, legal, military, research... Budget allocation for a missile interception system or for vaccine research units are two examples. Another example is the division of damage in an uncertain environment. ${ }^{1}$ And actors could also be factors, like for instance the pathologies that lead particular diseases or the circumstances that lead to a plane crash.

Hou et al. (2018) have translated this problem into a transferable utility game assuming that a coalition succeeds if at least one of its members reaches the target. They then apply the Shapley value to allocate the collective probability of success among the players. They propose an axiomatization of the Shapley value which turns out to be incorrect.

The probability game introduced by Hou et al. (2018) is concave and its Shapley value allocates to a player a share in the collective probability of success that is proportional to his individual probability of success. Furthermore, the coefficient of proportionality is anonymous, being defined by a playerindependent and symmetric function. We show that, together with symmetry (equal treatment of equals), proportionality characterizes the Shapley value on the class of probability games.

We consider other scenarios. Assuming that a coalition is successful if at least one of its members succeeds while the outside players all fail, the associated game is dual to the probability game as defined by Hou et al. This game is then convex and, the Shapley value being a self-dual allocation rule, it leads to the same allocation. Assuming that a coalition succeeds if only the member with the highest probability of success acts and reaches the target, the associated game is an airport game. It is a concave game whose Shapley value is well known and for which proper axiomatizations exist. We do not retain the game that results from assuming that a coalition succeeds if all its members succeed. The associated game is subadditive but its Shapley value may contain negative components.

Probability games have a connection with the existing literature on sharing the revenue that results from joint actions aiming at increasing the collective probability of success. For instance, Pickard et al. (2011) report on an experience of social mobilization where large groups of agents are recruited in order to contribute to the completion of a particular task in a minimum time. Hougaard et al. (2022) study models of evolving hierarchies where agents invest in recruiting additional agents in order to realize a particular task, like for instance the social mobilization just mentioned, mining in blockchains (Schrijvers et al. 2017), multilevel marketing (Emek et al. 2011) or prediction markets (Arrow et al. 2008).

The paper is organized as follows. Probability games and their duals are introduced in Section 2. Probability games have a nonempty core. It is the object of Section 3. Harsanyi dividends associated with probability games are defined and used in Section 4 to construct the Shapley value. Its axiomatization is the object of Section 5. Section 6 is devoted to the remaining scenarios and the last section offers concluding remarks.

\footnotetext{
${ }^{1}$ The apportionment of damage resulting from the actions of several tortfeasors is a problem studied by Dehez and Ferey (2013) and further investigated in Ferey and Dehez (2016).
} 


\section{Probability games}

A game with transferable utility is a pair $(N, v)$ where $N$ is the set of players and $v$ is a (characteristic) function that associates a real number to each coalition, its worth. By convention, $v(\varnothing)=0$. There are different ways to interpret the worth of a coalition. It may measure the payoff the coalition $S$ should or could obtain if it forms. ${ }^{2}$ We denote by $G$ the set of all games and by $G(N)$ the set of all games defined on the set $N$ of players.

Notation: Finite sets are denoted by upper-case letters. Lower-case letters are used to denote their cardinals: $t=|T|, s=|S|, \ldots$ For a vector $x, x(S)$ denotes the sum of its coordinates over the subset $S$. By convention, a sum over an empty set is zero. Set inclusion (non-strict) is denoted by $\subset$. We define $\mathcal{C}(S)=\{T \subset S \mid T \neq \varnothing\}$ and $\mathcal{C}_{i}(S)=\{T \subset S \mid i \in T\}$. Braces are sometimes omitted for coalitions: $v(i)$ replaces $v(\{i\}), v(\{i, j\})$ replaces $v(i, j), \ldots$

Consider a situation involving a set $N$ of players. They have a target to reach and each one is identified by his probability of reaching the target, $p_{i}>0$ for player $i$. Without loss of generality, we order the probabilities and we define $P_{n}=\left\{p \in[0,1]^{n} \mid 0<p_{1} \leq p_{2} \leq \ldots \leq p_{n} \leq 1\right\}$. A pair $(N, p)$ is a probability situation.

The question is the following. How to measure the contribution of each player to the collective probability of success, assuming probabilistic independence.

Consider a probability situation $(N, p), p \in P_{n}$. Assuming that a coalition is successful if at least one of its members reaches the target, the probability that the coalition succeeds is given by: ${ }^{3}$

$$
v(S)=1-\prod_{i \in S}\left(1-p_{i}\right)=\sum_{T \in \mathcal{C}(S)}(-1)^{t-1} \prod_{j \in T} p_{j} .
$$

This is the game introduced by Hou et al. (2018). ${ }^{4}$ Notice that $v(i)=p_{i}$ and $v(S)=1$ if (and only if) $p_{i}=1$ for some $i \in S$. In the 3-player case, we have:

$$
\begin{aligned}
& v(i)=p_{i} \quad i \in\{1,2,3\}, \\
& v(i, j)=1-\left(1-p_{i}\right)\left(1-p_{j}\right)=p_{i}+p_{j}-p_{i} p_{j} \quad i, j \in\{1,2,3\}, i \neq j, \\
& v(1,2,3)=1-\left(1-p_{1}\right)\left(1-p_{2}\right)\left(1-p_{3}\right)=p_{1}+p_{2}+p_{3}-p_{1} p_{2}-p_{1} p_{3}-p_{2} p_{3}+p_{1} p_{2} p_{3} .
\end{aligned}
$$

Example 1 The 3-player game associated to the probability vector $p=(0.3,0.5,0.7)$ is given by $v=(0.3,0.5,0.7|0.65,0.79,0.85| 0.895)$.

Example 2 The 4-player game associated to the probability vector $p=(0.2,0.4,0.5,0.7)$ is given by $v=(0.2,0.4,0.5,0.7|0.52,0.6,0.76,0.7,0.82,0.85| 0.76,0.856,0.88,0.91 \mid 0.928)$.

Interestingly, the dual game $\left(N, v^{d}\right)$ defined by $v^{d}(S)=v(N)-v(N \backslash S)$ is given by:

$$
v^{d}(S)=\prod_{j \in N \backslash S}\left(1-p_{j}\right)-\prod_{j \in N}\left(1-p_{j}\right)=\left(1-\prod_{j \in S}\left(1-p_{j}\right)\right) \prod_{j \in N \backslash S}\left(1-p_{j}\right) .
$$

\footnotetext{
${ }^{2}$ See Aumann (2010) who makes a distinction between what a coalition could claim vs what it could get, the latest referring implicitely to some underlying strategic form game.

${ }^{3}$ By convention, the product over an empty set is assumed to be equal to 1 .

${ }^{4}$ The wording that follows their definition points, however, to a different game: " $v(S)$ is the probability that all players $i$ in $S$ have a success, and all other players have a failure" (page 458).
} 
It is the probability that at least one player in $S$ succeeds, assuming that players outside $S$ all fail. It corresponds to the worst case: coalition $S$ should be allocated at least $v^{d}(S)$. The best case is instead given by $v_{1}(S)$ : coalition $S$ should not be allocated more. This is captured by the core.

Given an arbitrary game $(N, v)$ and a coalition $S \subset N$, the marginal contribution of player $i \in S$ to coalition $S$ is defined by $M C_{i}^{v}(S)=v(S)-v(S \backslash i)$. Two players $i$ and $j$ are substitutable if they contribute identically for all coalitions containing them: $M C_{i}^{v}(S)=M C_{j}^{v}(S)$ for all $S$ containing $i$ and $j$. A player $i$ is null if he never contributes: $M C_{i}^{v}(S)=0$ for all $S \subset N$.

A game is subadditive if $S \cap T=\varnothing \Rightarrow v(S \cup T) \leq v(S)+v(T)$. A game $(N, v)$ is concave (and thereby subadditive) if $v(S \cup T)+v(S \cap T) \leq v(S)+v(T)$ for all $S$ and $T$ in $N$. Equivalently, a game is concave if the marginal contributions of each player are decreasing with coalition size. ${ }^{5}$

The marginal contribution of player $i \in S$ to the probability of success of coalition $S$ is given by:

$$
M C_{i}^{v}(S)=p_{i} \prod_{j \in S \backslash i}\left(1-p_{j}\right) .
$$

It is the probability that player $i$ succeeds while all other members fail. Hence, two players $i$ and $j$ are substitutable if (and only if) $p_{i}=p_{j}$. In addition, marginal contributions are decreasing with respect to set inclusion:

$$
i \in S \subset T \Rightarrow p_{i} \prod_{j \in S \backslash i}\left(1-p_{j}\right) \geq p_{i} \prod_{j \in S \backslash i}\left(1-p_{j}\right) \prod_{j \in T \backslash S}\left(1-p_{j}\right)=p_{i} \prod_{j \in T \backslash i}\left(1-p_{j}\right) .
$$

Consequently, the game $(N, v)$ is concave and the dual game $\left(N, v^{d}\right)$ is convex.

\section{The core of probability games}

For an arbitrary game $(N, v)$, imputations are individually rational allocations $x \in \mathbb{R}^{n}$ satisfying the following inequalities: ${ }^{6}$

$$
v^{d}(i)=v(N)-v(N \backslash i) \leq x_{i} \leq v(i) \quad(i=1, \ldots, n) .
$$

The core of an arbitrary game $(N, v)$ is the set of coalitionally rational allocations:

$$
C(N, v)=\left\{x \in \mathbb{R}^{n} \mid x(N)=v(N) \text { and } x(S) \geq v(S) \text { for all } S \in \mathcal{C}(N)\right\}
$$

i.e. no coalition should receive less than its worth. ${ }^{7}$ However, under subadditivity, the appropriate definition of the core is the so-called anti-core defined by:

$$
A C(N, v)=\left\{x \in \mathbb{R}^{n} \mid x(N)=v(N) \text { and } x(S) \leq v(S) \text { for all } S \in \mathcal{C}(N)\right\}
$$

i.e. no coalition should contribute more than its worth. The anti-core of a game coincides with the core of its dual: $A C(N, v)=C\left(N, v^{d}\right)$.

Concavity ensures that the anti-core is the nonempty polyhedron whose vertices are the marginal contribution vectors: for any given players' ordering, players receive their marginal contribution following the order.

\footnotetext{
${ }^{5}$ A game $(N, v)$ is concave if the game $(N,-v)$ is convex. Convex games have been defined and studied by Shapley $(1971)$. ${ }^{6}$ Notice that the inequalities $v(N)-v(N \backslash i) \leq x_{i} \leq v(i)(i=1,2,3)$ completely define the (anti-) core for 3-player games.

${ }^{7}$ The idea of the core as the set of undominated allocations can be found in von Neumann and Morgenstern (1947, page 53). It was disregarded as a solution mainly because it is empty for zero-sum essential games. The term core was introduced by Gillies (1953) and was later introduced as an independent solution concept by Shapley (1955). See Zhao (2018) for a clarification of the respective contributions of Gillies and Shapley as to the core.
} 
Hence, for a probability situation $(N, p)$, imputations satisfy the following inequalities:

$$
p_{i} \prod_{j \in N \backslash i}\left(1-p_{j}\right) \leq x_{i} \leq p_{i} \quad(i=1, \ldots, n) .
$$

i.e. a player obtains at most his probability of success and at least the probability that he succeeds while all other player fails. Within the core, the ratio between what a coalition $S$ obtains and its worth satisfies the following inequalities:

$$
\prod_{j \in N \backslash S}\left(1-p_{j}\right) \leq \frac{x(S)}{v(S)} \leq 1 .
$$

Example 1 (continued) The anti-core of the probability game associated to the probability vector $(0.3,0.5,0.7)$ are defined by the following inequalities:

$$
\begin{aligned}
& 0.045 \leq x_{1} \leq 0.3, \\
& 0.105 \leq x_{2} \leq 0.5, \\
& 0.245 \leq x_{3} \leq 0.7 .
\end{aligned}
$$

\section{The Shapley value}

An allocation rule is a mapping $\varphi$ that associates an allocation $\varphi(N, v)$ to a game $(N, v)$. The requirement of efficiency is therefore built in the definition of allocation rule:

$$
\sum_{i \in N} \varphi_{i}(N, v)=v(N)
$$

Given a set of players $N$, the set $G(N)$ of all characteristic functions on $N$ is equivalent to the vector space $\mathbb{R}^{2^{n}-1}$. In proving the uniqueness of his value, Shapley (1953) shows that the collection of unanimity games $\left(N, u_{T}\right)$ defined by

$$
\begin{array}{rlrl}
u_{T}(S)=1 & & \text { if } T \subset S, \\
& =0 & & \text { if } T \not \subset S .
\end{array}
$$

forms a basis of $G(N)$ : for any given function $v \in G(N)$, there exists a unique $\left(2^{n}-1\right)$-dimensional vector $\alpha=\left(\alpha_{T}\right)_{T \in \mathcal{C}(N)}$ such that:

$$
v(S)=\sum_{T \in \mathcal{C}(N)} \alpha_{T} u_{T}(S)=\sum_{T \in \mathcal{C}(S)} \alpha_{T} .
$$

The $\alpha_{T}$ can be defined recursively, starting with $\alpha_{\varnothing}=0$, as follows:

$$
\alpha_{T}=v(T)-\sum_{\substack{S \in \mathcal{C}(T) \\ S \neq T}} \alpha_{S} \Rightarrow \alpha_{T}=\sum_{S \in \mathcal{C}(T)}(-1)^{t-s} v(S) \text { for all } T \subset N .
$$

Following Harsanyi (1959), $\alpha_{T}$ is the dividend accruing to coalition $T$ once all sub-coalitions have received their dividends. By (3), the sum of all dividends is equal to $v(N)$. An allocation can then be obtained by distributing the dividends of each coalition among its members. Harsanyi has shown that the Shapley value gives to each player the sum of the per capita dividend of the coalitions of which he is a member:

$$
S V_{i}(N, v)=\sum_{T \in \mathcal{C}_{i}(N)} \frac{1}{t} \alpha_{T}(N, v) \quad(i=1, \ldots, n) .
$$

Shapley (1953) proves that the value is the unique allocation rule $\varphi$ on the set of all games $G$ that satisfies symmetry, null player and additivity. Efficiency is built in the definition of allocation rule. 
Symmetry imposes equal treatment of substitutable players. Null player imposes a zero payoff to null players. Any reasonable allocation rule satisfies these three basic properties. The fourth, additivity, characterizes uniquely the Shapley value. It requires that the value of applied to a sum of games on a common set $N$ of players coincides with the sum of the values: $\varphi(N, v+w)=\varphi(N, v)+\varphi(N, w)$.

Young (1985) proposes an alternative axiomatization of the Shapley value in which additivity is replaced by a property of monotonicity. An allocation rule satisfies monotonicity if, considering two games $(N, v)$ and $(N, w)$ on a common set of players and a player $i \in N$,

$$
w(S)-w(S \backslash i) \geq v(S)-v(S \backslash i) \Rightarrow \varphi_{i}(N, w) \geq \varphi_{i}(N, v)
$$

i.e. the amount allocated to player $i$ is larger (or equal) in game $(N, w)$ than in game $(N, v)$ if the marginal contributions of player $i$ are larger (or equal) in game $(N, w)$. This implies that only the marginal contributions of a player enter into account in the computation of his share. Young proves that the Shapley value is the unique allocation rule that satisfies symmetry and monotonicity. ${ }^{8}$

Using (4), the Harsanyi dividends of the probability game $(N, v)$ associated to the probability vector $p \in P_{n}$ are given by:

$$
\alpha_{T}(N, v)=(-1)^{t-1} \prod_{i \in T} p_{i} .
$$

They alternate in sign according to coalition size and have no particular interpretation. The dividends of the dual game $\left(N, v^{d}\right)$ are given by:

$$
\alpha_{T}\left(N, v^{d}\right)=\prod_{j \in T} p_{j} \prod_{j \in N \backslash T}\left(1-p_{j}\right) .
$$

It is the probability that players in $T$ all succeed while players outside $T$ all fail. We observe that dividends of dual probability games are nonnegative. ${ }^{9}$

The Shapley value is a self-dual solution: the value of a game coincides with the value of its dual. Introducing successively (6) in (5) and (7) in (5), we obtain the two equivalent formulations of the Shapley value of the probability game associated to a probability situation $(N, p)$ :

$$
S V_{i}(N, p)=\sum_{T \in \mathcal{C}_{i}(N)} \frac{(-1)^{t-1}}{t} \prod_{j \in T} p_{j}=p_{i} \sum_{T \in \mathcal{C}_{i}(N)} \frac{(-1)^{t-1}}{t} \prod_{j \in T \backslash i} p_{j} \quad(i=1, \ldots, n),
$$

and

$$
S V_{i}(N, p)=\sum_{T \in \mathcal{C}_{i}(N)} \frac{1}{t} \prod_{j \in T} p_{j} \prod_{j \in N \backslash T}\left(1-p_{j}\right)=p_{i} \sum_{T \in \mathcal{C}_{i}(N)} \frac{1}{t} \prod_{j \in T / i} p_{j} \prod_{j \in N \backslash T}\left(1-p_{j}\right) \quad(i=1, \ldots, n) .
$$

Hence, whatever is the definition of the probability of success of a coalition, (1) or (2), the Shapley value defines the same allocation: (8) and (9) coincide. Hou et al. (2018) observe that the Shapley value allocates to a player a share that is proportional to his probability of success. The Shapley value can indeed be written as:

$$
S V_{i}(N, p)=p_{i} f\left(p_{-i}\right)
$$

\footnotetext{
${ }^{8}$ The null player axiom is not needed here. It is a consequence of monotonicity.

${ }^{9}$ Games whose dividends are nonnegative are positive games. They form an interesting class of convex games on which solution concepts tend to converge: the core coincides with the set of weighted Shapley values as well as the set of all distributions of Harsanyi dividends.
} 
where $p_{-i}$ denotes the vector of probabilities from which the coordinate $i$ has been removed and $f:[0,1]^{n-1} \rightarrow \mathbb{R}$ is a player-independent and symmetric function given by: ${ }^{10}$

$$
f(z)=1+\sum_{T \in \mathcal{C}(\{1, \ldots, n-1\})} \frac{(-1)^{t}}{t+1} \prod_{j \in T} z_{j} .
$$

In the 3-player case, player 1 obtains:

$$
S V_{1}(N, p)=p_{1}-\frac{1}{2}\left(p_{1} p_{2}+p_{1} p_{3}\right)+\frac{1}{3} p_{1} p_{2} p_{3}=p_{1}\left(1-\frac{1}{2}\left(p_{2}+p_{3}\right)+\frac{1}{3} p_{2} p_{3}\right)
$$

Example 1 (continued) The Shapley value of the game associated to the probability vector $(0.3,0.5,0.7)$ is given by $(0.155,0.285,0.455)$.

Example 2 (continued) The Shapley values of the game associated to the probability vector $(0.2,0.4,0.5,0.7)$ is given by $(0.088,0.192,0.251,0.397)$.

Concavity ensures that the Shapley value belongs to the anti-core.

As a consequence of (10), the share of a player in the collective probability is increasing with his probability of success. Furthermore, as shown by Hou et al. (Theorem 3.1), the following property holds: ${ }^{11}$

$$
p_{i} \geq p_{j} \Rightarrow S V_{i}(N, p) \geq S V_{j}(N, p) .
$$

Indeed, in the following difference, the expression between brackets is positive:

$$
S V_{i}(N, p)-S V_{j}(N, p)=\left(p_{i}-p_{j}\right)\left[1+\sum_{\substack{T \in \mathcal{C} N) \\ i, j \notin T}} \frac{(-1)^{t}}{t+1} \prod_{h \in T} p_{h}\right]
$$

Notice that (12) is actually an equivalence: $p_{i}>p_{j}$ if and only if $S V_{i}(N, p)>S V_{j}(N, p)$. This is a consequence of (13).

\section{Axiomatization of the Shapley value on the class of probability games}

We propose a simple axiomatization of the Shapley value restricted to probability games and expressed in terms of probabilities. Consider the following properties of an allocation rule $\varphi$ defined on the set games associated to probability vectors in $P_{n}$ :

A.1 Symmetry given any $p \in P_{n}, p_{i}=p_{j} \Rightarrow \varphi_{i}(N, p)=\varphi_{j}(N, p)$.

A.2 Proportionality given any $p \in P_{n}, \varphi_{i}(N, p)=\alpha_{i} p_{i}$ where $\alpha_{i}$ is independent of $p_{i}$.

These two properties characterize uniquely the Shapley value as an allocation rule on the class of probability situations.

Proposition 1 The Shapley value of a probability game $(N, v)$ is the unique allocation rule that satisfies A.1 and A.2. ${ }^{12}$

Proof Consider a probability situation $(N, p)$ and an allocation rule $\varphi$ satisfying A.1 and A2. According to A.2, there exist $n$ functions $f_{1}, \ldots, f_{n}$ such that $\varphi_{i}(N, p)=f_{i}\left(p_{-i}\right) p_{i}$ for all $i$. We first show that, under A1, the proportionality functions are independent of players' identities. We then

\footnotetext{
${ }^{10}$ A function is symmetric if permuting its arguments leaves its value unchanged.

11 This property is called "independent fairness" by Hou et al.

${ }^{12}$ Recall that efficiency is part of the definition of an allocation rule.
} 
show that the common proportionality function is uniquely defined and symmetric. As a result, $\varphi$ must be the Shapley value.

Consider a pair of players $(i, j)$ where $j=i+1$. For any given probability vector $p$ such that $p_{i}=p_{j}$, we have $p_{-i}=p_{-j}$ and $f_{i}\left(p_{-i}\right)=f_{j}\left(p_{-j}\right)$ by A.1. This applies for all $i=1, \ldots, n-1$. Independence then follows:

$$
\left.\left.f_{i}\left(z_{1}, \ldots, z_{n-1}\right)=f\left(z_{1}, \ldots, z_{n-1}\right) \text { for all }\left(z_{1}, \ldots, z_{n-1}\right) \in\right] 0,1\right]^{n-1} \text { and all } i \in N
$$

We now proceed iteratively to show that the function $f$ is uniquely defined, knowing that the probability to be allocated is a symmetric function of $p$ given by:

$$
F(p)=\sum_{T \in \mathcal{C}(N)}(-1)^{t-1} \prod_{i \in T} p_{i}=\sum_{i \in N} p_{i}-\sum_{i<j} p_{i} p_{j}+\sum_{i<j<k} p_{i} p_{j} p_{k}-\ldots
$$

We start by evaluating $f(z, \ldots, z)$ for some $z \in] 0,1]$ :

$$
n f(z, \ldots, z) z=F(z, \ldots, z) \Rightarrow f(z, \ldots, z)=\frac{1}{n z} F(z, \ldots, z) .
$$

In particular, for $n=2$, we have $f(z)=F(z, z) / 2 z$.

For $n \geq 3$, we proceed to the evaluation of $f\left(z_{1}, z, \ldots, z\right)$ for some $z_{1}$ and $\left.\left.z \in\right] 0,1\right]$ :

$$
f(z, \ldots, z) z_{1}+(n-1) f\left(z_{1}, z, \ldots, z\right) z=F\left(z_{1}, z, \ldots, z\right)
$$

or $f\left(z_{1}, z, \ldots, z\right)=\frac{1}{(n-1) z}\left(F\left(z_{1}, z, \ldots, z\right)-f(z, \ldots, z) z_{1}\right)$.

Next, we proceed to the evaluation of $f\left(z_{1}, z_{2}, z, \ldots, z\right)$ for $\left.\left.z_{1}, z_{2}, z \in\right] 0,1\right]$ :

$$
f\left(z_{2}, z, \ldots, z\right) z_{1}+f\left(z_{1}, z, \ldots, z\right) z_{2}+(n-2) f\left(z_{1}, z_{2}, z, \ldots, z\right) z=F\left(z_{1}, z_{2}, z, \ldots, z\right)
$$

or $f\left(z_{1}, z_{2}, z, \ldots, z\right)=\frac{1}{(n-2) z}\left(F\left(z_{1}, z_{2}, z, \ldots, z\right)-f\left(z_{2}, z, \ldots, z\right) z_{1}-f\left(z_{1}, z, \ldots, z\right) z_{2}\right)$.

We then continue to proceed similarly further until the evaluation of the complete function $f\left(z_{1}, z_{2}, z_{3}, \ldots, z_{n-1}\right)$ on $\left.] 0,1\right]^{n-1}$.

Notice that the resulting function is symmetric: all along the proof, the relative positions of $z_{1}, z_{2}, \ldots$ are arbitrary.

Here is an illustration of the proof for $n=4$. We start with the evaluation of $f(z, z, z)$ :

$$
f(z, z, z)=\frac{1}{4 z} F(z, z, z, z)=\frac{1}{4 z}\left(4 z-6 z^{2}+4 z^{3}-z^{4}\right)=1-\frac{3}{2} z+z^{2}-\frac{1}{4} z^{3} .
$$

For some $z_{1}$ and $\left.\left.z \in\right] 0,1\right]$, we have $f(z, z, z) z_{1}+3 f\left(z_{1}, z, z\right) z=F\left(z_{1}, z, z, z\right)$ or

$$
f\left(z_{1}, z, z\right)=\frac{1}{3 z}\left(F\left(z_{1}, z, z, z\right)-f(z, z, z) z_{1}\right)=1-z-\frac{z_{1}}{2}+\frac{z^{2}}{3}+\frac{2 z_{1} z}{3}-\frac{z_{1} z^{2}}{4} .
$$

For some $z_{1}, z_{2}$ and $\left.\left.z \in\right] 0,1\right]$, we have $f\left(z_{2}, z, z\right) z_{1}+f\left(z_{1}, z, z\right) z_{2}+2 f\left(z_{1}, z_{2}, z\right) z=F\left(z_{1}, z_{2}, z, z\right)$ or

$$
\begin{aligned}
f\left(z_{1}, z_{2}, z\right) & =\frac{1}{2 z}\left(F\left(z_{1}, z_{2}, z, z\right)-f\left(z_{2}, z, z\right) z_{1}-f\left(z_{1}, z, z\right) z_{2}\right) \\
& =1-\frac{z_{1}}{2}-\frac{z_{2}}{2}-\frac{z}{2}+\frac{z_{1} z_{2}}{3}+\frac{z_{1} z}{3}+\frac{z_{2} z}{3}-\frac{z_{1} z_{2} z}{4}
\end{aligned}
$$


It is the function defined in (11): the amount allocated by the Shapley value to player 1 is given by:

$$
S V_{1}(N, p)=p_{1} f\left(p_{2}, p_{3}, p_{4}\right)=p_{1}\left(1-\frac{1}{2}\left(p_{2}+p_{3}+p_{4}\right)+\frac{1}{3}\left(p_{2} p_{3}+p_{2} p_{4}+p_{3} p_{4}\right)-\frac{1}{4} p_{2} p_{3} p_{4}\right) .
$$

In their Theorem 3.3, Hou et al. (2018) propose an axiomatization of the Shapley value where they keep Shapley's original axioms, except for symmetry that they replace by independent fairness: an allocation rule $\varphi$ satisfies independent fairness if $v(i) \geq v(j) \Rightarrow \varphi_{i}(N, v) \geq \varphi_{j}(N, v) .{ }^{13}$ In their proof, they follow step-by-step the uniqueness proof originally proposed by Shapley (1953), a proof that applies to the class of all transferable utility games. Doing so is inappropriate in the context of probability games for the very simple reason that a sum of probability games is generally not a probability game: additivity cannot be invoked in this context. ${ }^{14}$

\section{Alternative scenarios}

Consider a probability situation $(N, p), p \in P_{n}$, and let us assume that the success of a coalition depends on the highest probability. The associated game is then given by:

$$
w(S)=\operatorname{Max}_{i \in S} p_{i}
$$

This is the well-known airport game introduced by Littlechild and Owen (1977). Only the context differs. The Shapley value of the max-game associated to the ordered probability vector $p \in P_{n}$ is given by:

$$
S V_{i}(N, p)=\sum_{k=1}^{i} \frac{p_{k}-p_{k-1}}{n-k+1}
$$

In the 3-player case, we have:

$$
S V(N, p)=\left(\frac{p_{1}}{3}, \frac{p_{1}}{3}+\frac{p_{2}-p_{1}}{2}, \frac{p_{1}}{3}+\frac{p_{2}-p_{1}}{2}+p_{3}-p_{2}\right)=\left(\frac{p_{1}}{3}, \frac{p_{2}}{2}-\frac{p_{1}}{6}, p_{3}-\frac{p_{2}}{2}-\frac{p_{1}}{6}\right) .
$$

Example 3 The max-game associated to the probability vector $p=(0.3,0.5,0.7)$ is given by $(0.3,0.5,0.7|0.5,0.7,0.7| 0.7)$ and its Shapley value is given by $(0.1,0.2,0.4)$.

Example 4 The max-game associated to the probability vector $p=(0.2,0.4,0.5,0.7)$ is given by $(0.2,0.4,0.5,0.7|0.4,0.5,0.7,0.5,0.7,0.7| 0.5,0.7,0.7,0.7 \mid 0.7)$ and its Shapley value is given by $(0.050,0.117,0.167,0.367)$.

Airport games are concave. Indeed, considering two coalitions $S$ and $T$ such that $w(S) \geq w(T)$, we have successively $w(S \cup T)=w(S)$ and $w(S \cap T) \leq w(T)$. As a consequence of concavity, the Shapley value of a max-game belongs to its anti-core.

Example 3 (continued) The anti-core of the max-game associated to the probability vector $(0.3,0.5,0.7)$ are defined by the following inequalities:

$$
\begin{aligned}
& 0 \leq x_{1} \leq 0.3, \\
& 0 \leq x_{2} \leq 0.5, \\
& 0.2 \leq x_{3} \leq 0.7 .
\end{aligned}
$$

\footnotetext{
${ }^{13}$ When applied to probability games, independent fairness actually implies symmetry.

${ }^{14} \mathrm{Hou}$ et al. actually impose linearity.
} 
Translated in the present context, Young's property of monotonicity simplifies as follows. Consider a player set $N$ and two probability vectors $p$ and $q$ in $P_{n}$. Young's property of monotonicity is equivalent to the following property:

A.3 Monotonicity Consider a player set $N$, a player $i \in N$ and probability vectors $p$ and $q$ in $P_{n}$. Then $q_{i} \geq p_{i}$ and $q_{i}-q_{j} \geq p_{i}-p_{j}$ for all $j<i \Rightarrow \varphi_{i}(N, q) \geq \varphi_{i}(N, p) .{ }^{15}$

Indeed, within the second scenario, the marginal contributions of player $i$ are of the form $p_{i}-p_{j}$ for all $j<i$, if any, while the marginal contribution of player $i$ to $\{i\}$ is equal to $p_{i}$. Monotonicity says that the amount allocated to player $i$ is larger (or equal) in the situation $(N, q)$ than in the situation $(N, p)$ if player $i$ has a larger probability of success and a larger probability differential with the players that precedes him.

Hence, symmetry and monotonicity characterize the Shapley value as an allocation rule on the class of max-games.

Proposition 2 The Shapley value of a max-game $(N, w)$ is the unique allocation rule that satisfies A.1 and A.3.

What about the scenario in which a coalition succeeds only if all its members reach the target? The associated characteristic function is then given by:

$$
w(S)=\prod_{i \in S} p_{i}
$$

It implies that acting together decreases the probability of success. This defines a subadditive game whose Shapley value may allocate negative amount to players with low probability of success. For example, the Shapley value resulting from the probability vector $p=(0.4,0.6,0.8)$ is given by $(-0.103,0.077,0.217)$. The same applies to other solutions concepts like for instance the nucleolus (Schmeidler, 1969). In case it applies to situations where each player is associated to an element in a chain, only the grand coalition is associated with a positive probability, a situation in which equal division is the only reasonable division.

\section{Concluding remarks}

Probabilistic independence has been assumed from the start. However, in many situations, actors or factors do not act independently. This is the case for instance when the factors are pathologies that can lead to death when combined. A characteristic function can still be constructed if the probabilities that any subset of players all succeed were known. Indeed, let us denote by $p_{i j}$ the probability that players $i$ and $j$ succeed, by $p_{i j k}$ the probability that players $i, j$ and $k$ succeed,... The probability that at least one player succeeds is then given by $F(p)=S_{1}-S_{2}+S_{3}-\ldots+(-1)^{n-1} S_{n}$ where

$$
S_{1}=\sum p_{i}, S_{2}=\sum \sum p_{i j}, S_{3}=\sum \sum \sum p_{i j k}, \ldots, S_{n}=p_{123 \ldots n} .16
$$

The Shapley value then retains the same structure but loses the property of proportionality. For instance, in the 3-player case, it allocates to player 1 an amount given by:

$$
S V_{1}(N, p)=p_{1}-\frac{1}{2}\left(p_{12}+p_{13}\right)+\frac{1}{3} p_{123} .
$$

\footnotetext{
${ }^{15}$ This is actually what has been proposed by Potters and Sudhölter (1999) with, however, a tiny difference: they impose a strict inequality sign on probability differences that is actually not needed.

${ }^{16}$ See Feller (1968, p. 99).
} 
Concerning the axiom of proportionality, it is remarkable that this simple property, together with symmetry, characterizes the Shapley value on the class of probability games: it is the property that differentiates the Shapley value from other symmetric allocation rules. Additivity does the same on the class of superadditive. Here, proportionality does not correspond to the Aristotelian definition of fairness: the ratio between the amounts allocated by the Shapley value does not coincide with the ratio between the individual probabilities. The particularity of probability games is that $p_{i}$ identifies player $i$ and knowledge of the probability vector $p$ is all what is needed to compute an allocation. By symmetry, we know that two players with the same probability of success get the same shares but the converse is also true:

$$
S V_{i}(N, p)=S V_{j}(N, p) \text { if and only if } p_{i}=p_{j} .
$$

This follows from (13). In addition, proportionality can be equivalently written as:

$$
p, q \in P_{n} \text { such that } p_{j}=q_{j} \text { for all } j \neq i \Rightarrow \frac{\varphi_{i}(N, p)}{\varphi_{i}(N, q)}=\frac{p_{i}}{q_{i}} \text {. }
$$

These observations justify proportionality.

The analysis in terms of a cooperative game of this probabilistic model is well defined when all probabilities are different from 1, possibly close to 1 . The results are formally compatible with a situation where a player has a probability 1 of success, in which case the collective probability of success is equal to 1 . If only one player has a probability 1 of success, should he be allocated one and zero to the other participants, independently of their probability of success? This is not what the Shapley value suggests simply because the other players are non-null. For example $(0.61,0.27,0.12)$ is the Shapley value associated to the probability vector $p=(0.3,0.6,1)$.

We must distinguish between two situations. Are we in an ex-ante or ex-post position? Ex-ante, we don't know and it is reasonable to assume that ignorance excludes a probability equal to one. On the other hand, an event that has a probability 1 of occurrence is not a sure event: it is almost sure. In both cases, granting a positive share to all players is justified. The problem differs in an ex-post situation. For example, someone has been shot to death and two persons have participated in the shooting, but only one fired the deadly bullet. In penal law, both would be sentenced, not necessarily to the same sentence. In civil law, a judge must determine who will pay and how much. Assuming that the shooter is a trained sniper, should he be the only one to contribute? Again, he could have missed the target so that both should be required to contribute. The Shapley value offers an apportionment basis.

\section{References}

Arrow K.J., et al. 2008. The promise of prediction markets. Science 320, 877-878.

Aumann, R.J., 2010. Some non-superadditive games, and their Shapley value, in the Talmud. International Journal of Game Theory 39, 3-10.

Dehez, P., Ferey, S., 2013. How to share joint liability: a cooperative game approach. Mathematical Social Sciences 66, 44-50.

Emek, Y., Karidi, R., Tennenholz, M., Zohar, A., 2011. Mechanisms for multi-level marketing. Proceedings of the 12th ACM conference on Electronic commerce, 209-218.

Feller, W., 1968. An introduction to probability theory and its applications (vol. 1, $3^{\text {rd }}$ edition). John Wiley \& Sons, New York. 
Ferey, S., Dehez, P., 2016. Multiple causation, apportionment and the Shapley value. Journal of Legal Studies 45, 143-171.

Gillies, D.B., 1953. Some theorems on n-person games. Ph.D. Thesis, Princeton University.

Harsanyi, J.C., 1959. A bargaining model for the cooperative n-person game. Tucker, A.W., Luce, D.R. (eds.) Contributions to the theory of games (Vol. IV), Annals of Mathematics Study 40, Princeton University Press, 325-355.

Hou, D., Xu, G., Sun, P., Driessen, T., 2018. The Shapley value for the probability game. Operations Research Letters 46, 457-461.

Hougaard, J.L., Moreno-Ternero, J.D., Østerdal L.P., 2022. Optimal management of evolving hierarchies. Management Science, 68, 6024-6038.

Littlechild, S.C., Owen G., 1973. A simple expression for the Shapley value in a special case. Management Science 20, 370-372.

Pickard, G., Pan, W., Rahwan, I., Cebrian, M., Crane, R., Madan, A., Pentland, A., 2011. Timecritical social mobilization. Science, 334 (6055), 509-512.

Potters, J., Sudhölter, P., 1999. Airport problems and consistent allocation rules. Mathematical Social Sciences 38, 83-102.

Roth, A.E. (Ed., 1988). The Shapley value. Essays in honor of Lloyd Shapley. Cambridge University Press, Cambridge, 1988.

Schmeidler, D., 1969. The nucleolus of a characteristic function game. SIAM Journal of Applied Mathematics 17, 1163-1170.

Shapley, L.S., 1953. A value for n-person games. Kuhn H., Tucker A.W. (eds.), Contributions to the Theory of Games II. Annals of Mathematics Studies 24, Princeton University Press, Princeton, 307317. Reproduced in Roth (Ed. 1988), 31-40.

Shapley, L.S., 1971. Cores of convex games. International Journal of Game Theory 1, 11-26.

Shrijvers, O., Boneh, D., Roughgarden, T., 2017. Incentive compatibility of bitcoin mining pool reward functions. Groossklags, J., Preneel, B. (eds.). Financial Cryptography and Data Security: FC2016. Lecture Notes on Computer Science 9603, 477-498.

von Neumann, J., Morgenstern O., 1944. Theory of games and economic behavior. Princeton University Press, Cambridge (latest edition dated 1953).

Young, P., 1985. Monotonic solutions of cooperative games. International Journal of Game Theory $14,65-72$.

Zhao, J., 2018. Three little-known and yet still significant contributions of Lloyd Shapley, Games and Economic Behavior 108, 592-599. 